\title{
The development of a quarantine strategy is an important path to a normalized response to COVID-19
}

\author{
Han $\mathrm{Zhu}^{1,2}$, Hongzhou Lu ${ }^{1, *}$ \\ ${ }^{1}$ Department of Infectious Diseases, Shanghai Public Health Clinical Center, Fudan University, Shanghai, China; \\ ${ }^{2}$ School of Public Health, Bengbu Medical College, Bengbu, Anhui, China.
}

SUMMARY The ongoing pandemic of coronavirus disease 19 (COVID-19) is still in a global pandemic that has affected more than 200 countries. When prevention and control of COVID-19 is gradually normalized, communication between countries needs to be gradually restored due to development needs. There are 34 vaccines in the clinical evaluation stage and 145 vaccines in the preclinical evaluation stage in the global COVID-19 vaccine research and development program, but the rate and process of vaccination may not be sufficient to meet the current needs of society for restoring development and communication. Studies have found that chloroquine, favipiravir, remdesivir and other drugs are useful for COVID-19, but currently there is no specific drug for the treatment of COVID-19. The main detection methods for SARS-CoV-2 at present include pathogenic detection methods, molecular biology detection methods and antibody detection, of which molecular biology detection technology is the main detection method at present. There are some more convenient and rapid detection methods. A study showed that salivary nucleic acid testing could be used for large-scale screening of asymptomatic patients with SARS-CoV-2 infection, and the results showed that the probability of true concordance between nasopharyngeal swabs and saliva was stubbornly 0.998 (90\% CI: 0.996-0.999). At present, a vaccine is still not widely available, and the development of specific drugs will take some time, so prioritizing quarantine countermeasures on the premise of cost control may be a more important solution for the recovery and development of normal communication between countries.

Keywords COVID-19, vaccines, specific drugs, quarantine countermeasures

\section{Status of the epidemic}

According to Worldometer data, as of October 9, Beijing time, a total of $36,706,900$ cases of coronavirus disease19(COVID-19) had been diagnosed worldwide, with a cumulative total of $1,065,646$ deaths and more than 10 thousand cases diagnosed in 105 countries. The first case of COVID-19 was detected in December 2019 (1), and the epidemic is still in a global pandemic that has affected more than 200 countries. It poses a serious threat to the life and health of the population of all countries and affects the normal social order and development. The mortality rate of COVID-19 varies significantly by age. With hospital mortality rates below $5 \%$ among patients under 40 years of age, $35 \%$ among patients aged 70-79 years, and over $60 \%$ among patients aged $80-89$ years (2). The epidemic has also caused a sharp decline in communication between countries, with the transnational flow of goods, people and services temporarily halted to reduce the spread of the neo-crown virus. With the gradual control of the epidemic, the prevention and control of COVID-19 will be gradually normalized, and the communication between countries will be gradually restored due to development needs.

\section{Control situation}

WHO concluded on March 10, 2020, that "the outbreak in China is ending". As of October 8, 21 new confirmed cases have been reported nationwide, all of which were imported from overseas (3), Figure 1. At present, the epidemic in China has gradually come under control, and in order to promote social development and international contacts as soon as possible, it is necessary to start from the three aspects of prevention, treatment and quarantine, in order to restore economics and communication while keeping the possibility of virus transmission to a minimum level.

\subsection{Vaccines}

According to World Health Organization (WHO) data 


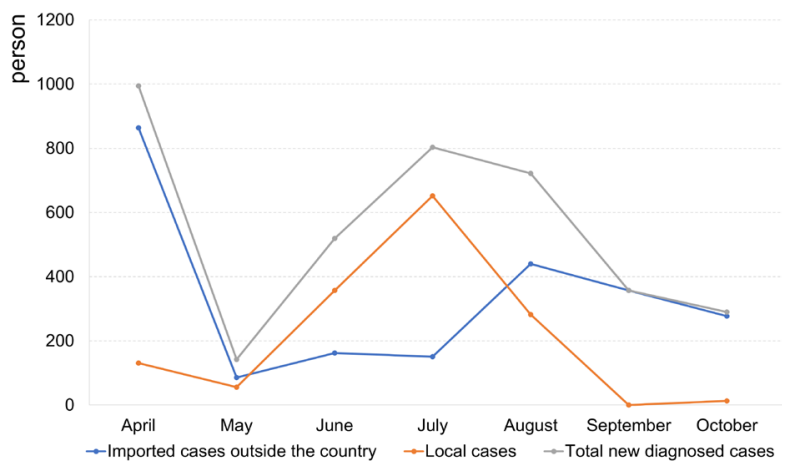

Figure 1. Trend of new cases in 2020. Local cases started to show an increasing trend in May and gradually decreased in July, while the number of imported cases from abroad gradually increased in July. At present, the new confirmed cases are mainly imported from overseas, and quarantine should be the mainstay of prevention and control to prevent overseas importation.

(4), as of September 8, 2020, there are 34 vaccines in the clinical evaluation stage and 145 vaccines in the preclinical evaluation stage in the global COVID-19 vaccine research and development program, and a total of four vaccines in China have entered phase III clinical trials. The results of the Phase II clinical trial of recombinant COVID-19 vaccine (adenovirus vector) developed by Chen Wei showed that $99.5 \%$ of subjects produced specific antibodies, 95.3\% produced neutralizing antibodies, and $89 \%$ produced specific T-cell immune reactions 28 days after a single vaccination, which can meet the technical requirements for emergency use (5). Although breakthroughs have been achieved, the rate and process of vaccination may not be sufficient to meet the current needs of society for restoring development and communication.

\subsection{New drugs and specific drugs}

There is no specific drug for the treatment of COVID-19. Studies have shown that chloroquine has an inhibitory effect on severe acute respiratory syndrome coronavirus 2 (SARS-CoV-2) (6), and its derivative chloroquine phosphate has been recommended for the antiviral treatment of COVID-19. Some studies have found that in vitro application of favipiravir significantly inhibits COVID-19 and accelerates viral clearance (67). Favipiravir and its generics have been included in treatment protocols in India and Russia respectively (8$9)$. Remdesivir is an antiviral drug developed for the Ebola virus, and was found to have anti- SARS-CoV-2 effects when used in vitro (6), and a clinical trial in the United States showed that remdesivir shortened recovery time in hospitalized patients with SARS-CoV-2 infection of the lower respiratory tract (10). Although effective antiviral drugs have been developed by targeting viral proteases, polymerases and host proteins in the Middle East Respiratory Syndrome and SARS epidemics, there are no specific antiviral drugs for SARS-CoV-2.

\subsection{Detection technology}

The rapid and accurate detection of SARS-CoV-2 is an important tool to control the spread of the epidemic, and the main detection methods for SARS-CoV-2 at present include pathogenic detection methods, molecular biology detection methods and antibody detection, of which molecular biology detection technology is the main detection method at present. CRISPR-based assays and flow-immunochromatography have the advantages of rapid testing, low cost and high sensitivity, which can be used for field testing (11). Due to the persistence and extensiveness of the epidemic, continuous investment and testing have been carried out to continuously update the detection technology and explore more convenient and rapid detection methods. The University of Helsinki, Finland, conducted a SARS-CoV-2 test using the odor discrimination ability of dogs (12), which showed that 10 screened dogs could accurately identify neocrownpositive odors. The first batch "COVID-19 Detection Dogs" are now in use at Finnish airports, where they can detect an abnormality by sniffing the wipes on the arms of test subjects, which takes only 10 seconds. Takanori Teshima et al. (13) showed that salivary nucleic acid testing could be used for large-scale screening of asymptomatic patients with SARS-CoV-2 infection. 1924 people were included in the study to compare the sensitivity and specificity of SARS-CoV-2 nucleic acid testing of nasopharyngeal swabs and saliva, and the results showed that the probability of true concordance between nasopharyngeal swabs and saliva was stubbornly 0.998 (90\% CI: 0.996-0.999), with a high correlation between nasopharyngeal swabs and saliva for SARS-CoV-2 load in SARS-CoV-2 nucleic acidpositive patients. Quarantine countermeasures are of great practical importance due to the specificity of the exchange situation under the normalized demands of social development.

In conclusion, the full-blown COVID-19 epidemic has affected countries in many ways, but there is still a developmental need in society. At present, the vaccine is still not widely available, and the development of specific drugs will take some time, so the rapid detection of SARS-CoV-2 infection in asymptomatic people is the key to prevent and control the outbreak of SARS-CoV-2 epidemic, and prioritizing quarantine countermeasures on the premise of cost control may be a more important solution for the recovery and development of normal communication between countries. Therefore, while developing vaccines and specific drugs, quarantine countermeasures should be explored under the premise of cost control.

Funding: This study was funded by Efficacy and Safety of Hydroxychloroquine Sulfate in Treating Pneumonia Caused by Novel Coronavirus Infection from Shanghai Municipal Science and Technology Commission 
(20431900103); Research on Clinical Diagnosis and Treatment Standards of Pneumonia with Novel Coronavirus Infection from Fudan University.

Conflict of Interest: The authors have no conflict of interest to disclose.

\section{References}

1. Malik YS, Sircar S, Bhat S, Sharun K, Dhama K, Dadar $\mathrm{M}$, Tiwari R, Chaicumpa W. Emerging novel coronavirus (2019-nCoV)-current scenario, evolutionary perspective based on genome analysis and recent developments. Vet Q. 2020; 40:68-76.

2. Richardson S, Hirsch JS, Narasimhan M, et al. the Northwell COVID-19 Research Consortium. Presenting characteristics, comorbidities, and outcomes among 5700 patients hospitalized with COVID-19 in the New York City area. JAMA. 2020; 323:2052-2059.

3. National Health Commission of the People's Repulic of China. An update of Novel Coronavirus Pneumonia break out as of 24:00 on 9 October. http://www.nhc.gov.cn/xcs/ yqtb/202010/b4298767b6104a80a15ec7e8e477e322.shtml (accessed October 14, 2020). (in Chinese)

4. World Health Organization. Timeline of WHO's response to COVID-19, Last updated 9 September 2020. https:// www.who.int/news/item/29-06-2020-covidtimeline (accessed October 14, 2020).

5. Zhu FC, Wurie AH, Hou LH, et al. Safety and immunogenicity of a recombinant adenovirus type-5 vector-based Ebola vaccine in healthy adults in Sierra Leone: a single-centre, randomised, double-blind, placebocontrolled, phase 2 trial. Lancet. 2017; 389:621-628.

6. Wang M, Cao R, Zhang L, Yang X, Liu J, Xu M, Shi Z, Hu Z, Zhong W, Xiao G. Remdesivir and chloroquine effectively inhibit the recently emerged novel coronavirus (2019-nCoV) in vitro. Cell Res. 2020; 30:269-271.

7. Cai Q, Yang M, Liu D, et al. Experimental Treatment with Favipiravir for COVID-19: An Open-Label Control Study. Engineering (Beijing). 2020; doi: 10.1016/ j.eng.2020.03.007.

8. Russian Direct Investment Fund. RDIF and ChemRar Announce Increased Production of Avifavir for Treatment of Coronavirus and Begin Export Deliveries. https://www. prnewswire.com/ae/news-releases/rdif-and-chemrarannounce-increased-production-of-avifavir-for-treatmentof-coronavirus-and-begin-export-deliveries-832625464. html (accessed October 14, 2020).

9. Glenmark gets India approval for favipiravir as Covid-19 treatment. https://www.hindustantimes.com/businessnews/glenmark-gets-india-approval-for-favipiravir-ascovid-19-treatment/story-nfUZfzToLISJa2LEE7lsbK.html (accessed October 14, 2020).

10. Paladugu S, Donato AA. Remdesivir improved time to recovery in adults hospitalized with COVID-19 and lower respiratory tract involvement. Ann Intern Med. 2020; 173:JC4.

11. Gootenberg JS, Abudayyeh OO, Lee JW, et al. Nucleic acid detection with CRISPR- Cas $13 \mathrm{a} / \mathrm{C} 2 \mathrm{c} 2$. Science. 2017; 356:438-442.

12. Daily Mail. Meet British dogs trained sniff coronavirus airports. https://www.dailymail.co.uk/femail/ article-8770311/Meet-British-dogs-trained-sniffcoronavirus-airports.html (accessed October 14, 2020).

13. Yokota I, Shane PY, Okada K, Unoki Y, Yang Y, Inao T, Sakamaki K, Iwasaki S, Hayasaka K, Sugita J, Nishida M, Fujisawa S, Teshima T. Mass screening of asymptomatic persons for SARS-CoV-2 using saliva. Clin Infect Dis. 2020; ciaa1388. doi: 10.1093/cid/ciaa1388.

Received October 2, 2020; Revised October 20, 2020; Accepted October 22, 2020.

\section{*Address correspondence to:}

Hongzhou Lu, Department of Infectious Diseases, Shanghai Public Health Clinical Center, Fudan University, 2901 Caolang Road, Jinshan District, Shanghai 201508, China.

E-mail: luhongzhou@fudan.edu.cn

Released online in J-STAGE as advance publication October 25, 2020. 